\title{
Commentary \\ Is tissue Doppler echocardiography the Holy Grail for the intensivist?
}

\author{
Jan Poelaert and Carl Roosens
}

University Hospital Ghent, Department of Intensive Care Medicine and Cardiac Anaesthesia, 5 K12 IE, De Pintelaan 185, B-9000 Gent, Belgium

Corresponding author: Jan Poelaert, jan.poelaert@ugent.be

Published: 6 June 2007

This article is online at http://ccforum.com/content/11/3/135

(c) 2007 BioMed Central Ltd

See related research by Vignon et al., http://ccforum.com/content/11/2/R43
Critical Care 2007, 11:135 (doi:10.1186/cc5903)

\begin{abstract}
Assessment of left ventricular diastolic function in the critically ill patient remains a difficult issue in clinical practice. Combined use of routine transmitral and pulmonary venous Doppler patterns in conjunction with tissue Doppler imaging have been claimed to allow bedside diagnosis of diastolic dysfunction. Although in the previous issue of Critical Care it was clearly demonstrated there might be a difference in load dependency of the early myocardial tissue Doppler velocity between lateral and septal placed sample volume, there remain still several unanswered questions, particularly with respect to the preload dependency of these indices.
\end{abstract}

The clinical evaluation of left ventricular (LV) diastolic function has been a difficult challenge. LV filling and pulmonary venous Doppler patterns have been utilized to estimate LV diastolic function, comprising variables as such stiffness, relaxation and even LV filling pressures. Doppler echocardiography has been utilized not only as a diagnostic tool but also as a monitoring tool, permitting follow-up of the effects of therapeutic interventions. In the previous issue of Critical Care, Vignon and colleagues demonstrated the value of tissue Doppler imaging (TDI) to estimate LV diastolic function in a setting where acute alterations of preloading conditions may interfere [1]. They also clearly show some weak points with respect to the correct use of these function variables.

A typical transmitral Doppler pattern consists of a larger early filling velocity wave ( $E$ wave) followed by an atrial contraction flow velocity wave. Reduced LV relaxation (present in patients with advanced age, ischaemic heart disease or arterial hypertension) will induce a reduction of the $E$ wave in comparison with the atrial contraction flow velocity wave. Increasing filling pressures will increase the $E$ wave velocity and will shorten the deceleration time of the $E$ wave, with a transition from pseudonormalization to a restrictive pattern
[2,3]. Concomitantly, the pulmonary venous Doppler pattern will change accordingly. Determining the phase of diastolic dysfunction for an individual patient remains difficult because of the interplay between relaxation and preload, which makes the routine pulsed wave Doppler indices useless - particularly in a setting where preloading conditions may change constantly and abruptly, as in many critically ill patients.

TDI could help in discriminating the phase of diastolic dysfunction [4]. This Doppler mode facilitates the assessment of the movement of the myocardial tissue, which typically reflects low velocity with very high amplitude Doppler signals. TDI can be obtained by either spectral or colour Doppler techniques. TDI can therefore be utilized to estimate myocardial velocities at the mitral annulus to obtain an impression of both systolic and diastolic myocardial motion. The spectral Doppler pattern is characterized by a systolic wave, an early diastolic wave ( $E^{\prime}$ wave) and an atrial velocity wave [5]. The technique is hampered by shortcomings related to the Doppler technology (angle misalignment, translation and rotation of the myocardial tissue) and by intrinsic characteristics of myocardial function (for example, the presence of regional wall motion abnormalities). Nevertheless, regional LV systolic function and LV diastolic function assessment is possible provided the sample volume is placed at the level of the mitral annulus and no ischaemia/ infarction in the annulus region is present. Whereas the systolic component of this Doppler pattern has been shown to be clearly preload dependent [5], less transparency exists on the load dependency of the diastolic myocardial velocities. With the initial description of this variable, the index appeared to be load independent in settings of rapid infusion or preload alteration. Jacques and colleagues, however, reported both afterload independency and preload dependency of the $E^{\prime}$ wave in situations where a normal LV function was present

$E$ wave = early filling velocity wave; $E^{\prime}=$ early diastolic wave; $L V=$ left ventricular; $T D I=$ tissue Doppler imaging. 
[6]. In contrast to the present study of Vignon and colleagues [1], Jacques and colleagues did not report the sample volume location. Comparison between the septal and lateral mitral annulus signals suggests that the septal signals are apparently more sensitive to preload alterations. The reader, however, should bear in mind that the stability of the signals obtained at the septal side can be questioned, particularly in mechanically ventilated patients with ventilation-induced, right-sided, afterload shifts [7,8]. Nevertheless, several authors have already reported differences in $E^{\prime}$ wave velocities between the septal and lateral walls, without the ability to indicate a clear cause for this difference.

Vignon and colleagues show no changes of the $E^{\prime}$ wave in a rather small subset of intensive care unit patients, and again several issues have to be kept in mind. First, several of the study patients were supported by a vasopressor. The effects of drugs such as epinephrine, however, have not been described; one should expect an increase of the $E^{\prime}$ wave, at least when considering a similar effect to that with dobutamine [9]. It can thus be questioned whether an ultrafiltration (and thus load diminishing) effect is not concurring, keeping the $E^{\prime}$ wave constant. Second, the influence of ultrafiltration itself on LV systolic function and LV diastolic function during septic shock is largely unknown. The combination of lower preload (by ultrafiltration) and decreased LV function would render a less preload-dependent $E^{\prime}$ wave, as suggested by Jacques and colleagues [6] The number of intensive care unit patients included in this subset is again far too small to conclude in a proper manner.

From all these studies, it is unclear whether the discussed concepts apply to all haemodynamic states. The fact that the $\mathrm{E}$ wave/E' wave relates to pulmonary capillary wedge pressure [10] suggests that increased intrathoracic pressures could impede this relationship strongly. This question also remains open and unanswered. Apart from the fact that the $E^{\prime}$ wave (in conjunction with traditional Doppler parameters) is useful in determining LV diastolic function more accurately, TDI in the critically ill patient keeps more questions open than are answered.

\section{Competing interests}

The author(s) declare that they have no competing interests.

\section{Acknowledgement}

This work was supported by an unrestricted grant from the International Research Centre of Ghent University.

\section{References}

1. Vignon $\mathrm{P}$, Allot V, Lesage J, Martaillé J-F, François B, Gastinne H: Diagnosis of left ventricular diastolic dysfunction in the setting of acute changes in loading conditions. Crit Care 2007, 11:R43.

2. Nishimura R, Abel M, Hatle L, Tajik A: Relation of pulmonary vein to mitral flow velocities by transesophageal Doppler echocardiography. Effect of different loading conditions. Circulation 1990, 81:1488-1497.
3. Nishimura RA, Miller Jr FA, Callahan MJ: Doppler echocardiography: theory, instrumentation, technique and application. Mayo Clin Proc 1985, 60:321-343.

4. Sohn D-W, Chai I-H, Lee D-J, Kim H-C, Kim H-S, Oh B-H, Lee MM, Park Y-B, Choi Y-S, Seo J-D, et al.: Assessment of mitral annulus velocity by Doppler tissue imaging in the evaluation of left ventricular diastolic function. J Am Coll Cardiol 1997, 30:474-480.

5. Amà R, Segers $P$, Roosens $C$, Claessens $T$, Verdonck P, Poelaert $\mathrm{J}$ : Effects of load on systolic mitral annular velocity by tissue Doppler imaging. Anesth Analg 2004, 99:332-338.

6. Jacques DC, Pinsky MR, Severyn D, Gorcsan J, III: Influence of alterations in loading on mitral annular velocity by tissue Doppler echocardiography and its associated ability to predict filling pressures. Chest 2004, 126:1910-1918.

7. Poelaert J, Visser C, Everaert J, De Deyne C, Decruyenaere J, Colardyn F: Doppler assessment of right ventricular outflow impedance during positive pressure ventilation. J Cardiothorac Vasc Anesth 1994, 8:392-397.

8. Roosens $\mathrm{C}$, Amà R, Leather $\mathrm{H}$, Segers $\mathrm{P}$, Sorbara $\mathrm{C}$, Wouters $\mathrm{P}$, Poelaert J: Hemodynamic effects of different lung protective ventilation strategies in closed chest pigs with normal lungs. Crit Care Med 2006, 34:2990-2996.

9. von Bibra H, Tuchnitz A, Klein A, Schneider-Eicke J, Schomig A, Schwaiger M: Regional diastolic function by pulsed Doppler myocardial mapping for the detection of left ventricular ischemia during pharmacologic stress testing: a comparison with stress echocardiography and perfusion scintigraphy. J Am Coll Cardiol 2000, 36:444-452.

10. Nagueh S, Middleton K, Kopelen H, Zoghibi W, Quinones M: Doppler tissue imaging: a non-invasive technique for evaluation of left ventricular relaxation and estimation of filling pressures. J Am Coll Cardiol 1997, 30:1527-1533. 